\title{
STRUCTURE DETECTION WITH SECOND ORDER RIESZ TRANSFORMS
}

\author{
Dascha Dobrovolskij ${ }^{1,2}$, Johannes Persch ${ }^{3}$, KATJa Schladitz ${ }^{2}$ And Gabriele \\ $\mathrm{STEIDL}^{\otimes, 3}$ \\ ${ }^{1}$ Fraunhofer ITWM, Fraunhofer-Platz 1, D-67663 Kaiserslautern, Germany; ${ }^{2}$ Hochschule Darmstadt, \\ Fachbereich Mathematik und Naturwissenschaften, Schöfferstraße 3, D-64295 Darmstadt, Germany; \\ ${ }^{3}$ Department of Mathematics, Technische Universität Kaiserslautern, Paul-Ehrlich-Str. 31, 67663 Kaiserslautern, \\ Germany \\ e-mail: steidl@mathematik.uni-kl.de
}

(Received June 21, 2018; revised October 31, 2018; accepted November 23, 2018)

\begin{abstract}
A frequently applied indicator of tubular structures is based on the eigenvalues of the Hessian matrix of the original image convolved with a Gaussian, whose standard derivation depends on the size of the tubes. Hence the tube size must either be known in advance or a whole scale of standard deviations has to be tested resulting in higher computational costs - a serious obstacle for data with varying tube thickness.

In this paper, we propose to modify the structure indicator by replacing the derivatives of the Gaussian smoothed function by the Riesz transform. We show by various numerical examples that the resulting structure indicator is scale independent. Smoothing with a Gaussian is just necessary to cope with the noise in the image, but is not related to the size of the tubular structures. We apply the novel structure indicator for the fiber orientation analysis of fibrous materials and for the segmentation of leather. The latter one was a special challenging application since all scales are present in the microstructure of leather.
\end{abstract}

Keywords: image analysis, local fiber orientation, tubular structures.

\section{INTRODUCTION}

The motivation of this paper came from the practical task to analyze the 3D microstructure of felt and bovine leather. In general, the detection of structures in data sets of two or three dimensions is an important task in image processing. These structures can be used as features in computer vision or to deduce statistical properties of the data set. Simple structure tensors as those of Förstner and Gülch (1987), focus on the detection of edges and vertices. Incorporating higher order derivatives as in the boundary tensor by Köthe (2003) improves the detection or as in the structure tensor of Aach et al. (2006), see also Steidl and Teuber (2009), the direction of overlapping structures and at occlusions can be found. Further, more robust energy tensor for edge detection were constructed by Felsberg and Granlund (2004). Using combinations of higher order derivatives, more complex features such as ridges or frequencies of textures can be detected, see Lindeberg (1999) for an overview.

In many applications it is essential to isolate certain constituents, e.g., fibers, blobs or plates. Blood vessels can be seen in magnetic resonance angiography, but their detection is difficult due to the presence of other organs. Therefore an enhancement of the blood vessels is of interest, see Prince (1994), Du et al. (1995), and Frangi et al. (1998). Frangi et al. (1998) use the eigenvalues of the Hessian matrix of the data convolved with a Gaussian function to distinguish vessels from other constituents where the parameter $\sigma$ of the Gaussian function determines the scale. In particular, they interprete properties on the eigenvalues of Gauss Hessian matrices for the occurrence of fibers, plates, and blobs. The main drawback of the Gauss Hessian matrix as structure tensor is its dependence on the scale of the underlying structure. One way to overcome this dependence is scanning the image for all scales of interest and taking the maximal response obtained over the scales as final result. Still this does not solve the problem completely, as we need to calculate the eigenvalues of the Gauss Hessian matrix for each scale separately leading to a high computational effort and the computation of the large scales comes along with blurring of small gaps between structures.

Besides detecting locations of structures, the estimation of their local directions is of interest. Several methods have been proposed by Robb et al. (2007); Altendorf and Jeulin (2009); Krause et al. (2010); Redenbach et al. (2012) that do not require the segmentation of individual fibers. The concise comparison by Wirjadi et al. (2016) shows that the two methods based on first (Krause et al., 2010) or second (Redenbach et al., 2012) order derivatives - the 
structure tensor and the Gauss Hessian matrix - yield comparable results. Moreover, they are more precise than the competitors investigated by Wirjadi et al. (2016) requiring a discretization of the orientation space, i.e., the maximum response of anisotropic Gaussians by Wirjadi et al. (2009) and the inertia method of Altendorf and Jeulin (2009).

The local fiber orientation analysis based on the eigenvalue analysis of the Gauss Hessian matrix has been successfully applied for various materials, e.g., by Heieck et al. (2017); Sliseris et al. (2016); Wirjadi et al. (2014). However, application of this method requires the fiber thickness as an input parameter. More precisely, the fiber thickness is needed in order to properly choose the parameter $\sigma$ of the Gaussian filter applied to the gray value image for subsequent calculation of the Hessian. This is very often known as in the case of glass fibers. In other cases, rough estimates can be derived from the image data. Nevertheless, for data with varying fiber thickness, even if these are known, this requirement represents a serious obstacle.

In this paper, we propose to replace the Gauss Hessian matrix by a Riesz Hessian matrix and to use the Riesz Hessian within the simple structure measures proposed by Frangi et al. (1998). Instead of smoothing the data with a Gaussian of appropriate size and taking derivatives, we approximate the derivatives by the Riesz transform (1928). The Riesz transform as a generalization of the Hilbert transform (Stein and Weiss, 1971) is known to be scale independent (Unser and Van De Ville, 2010) and closely related to the gradient. Smoothing with a Gaussian of small fixed size is still necessary to cope with noisy data, but a scale adaptation of the Gaussian is superfluous. The application of the Riesz transform within structure location and orientation detection is not new. So the Riesz transform was used within Aach's structure tensor by Sedlazeck (2008) and within Köthe and Felsberg's boundary tensor 2005. The monogenic signal (Felsberg and Sommer, 2001), which consists of the function itself and its Riesz transform, together with sophisticated multiscale techniques was applied as structure detector, e.g., by Unser and Van De Ville (2010); Storath (2011); Häuser et al. (2014). However, the application of the Riesz transform within the Hessian together with the structure measures from Frangi et al. (1998) was not reported in the literature so far. For an optical filter point of view of the Riesz transform we refer to Larkin et al. (2001); Schausberger et al. (2010).

We propose to apply this novel structure descriptor to real-world data containing structures at different scales: Glass and carbon fiber reinforced composites are attractive light weight materials having applications for example in the automotive, aerospace and wind energy industries. The mechanical properties of components made from fiber reinforced polymers are crucially influenced by spatial distribution and orientation of the reinforcing fibers within the component. For complex shaped components, the final local fiber orientation is hard to control. Dedicated numerical flow simulation tools are used to predict fiber orientations for given design of the part under consideration and production parameters. Micro-computed tomography $(\mu \mathrm{CT})$ and subsequent quantitative analysis of the resulting 3D images are the method of choice for validating simulation results by comparison with real observations, see, e.g., Wonisch and Wüst (2014).

Recently, the 3D microstructure of leather has attracted increasing interest. Non-destructive testing methods like ultrasound imaging, small angle $\mathrm{X}$ ray scattering, and $\mathrm{CT}$ have been applied to capture structural features of the collagen fiber bundles like orientation, density or connectivity, see, e.g., BasilJones et al. (2010), Bittrich et al. (2014), and Wells et al. (2016). Bittrich et al. (2014) showed that the quantitative analysis of $\mu \mathrm{CT}$ images of leather microstructures is possible based on a binarization. That is, valuable structural information can be gained by a segmentation into collagen fiber bundle structure (foreground) and pore space (background). Finite element simulations of mechanical properties of leather based on the microstructure require however to segment individual structural elements of the leather's microstructure. That is, the dense and strongly interwoven bundles have to be split into rather simply shaped elements while preserving their connectivity relations. This task is very demanding due to the multiscale nature of the leather's microstructure and the fact, that the scales are not clearly separated, see Godehardt et al. (2017) for more details and Fig. 1. Moreover, being a natural material, the structure varies strongly. Hence it is necessary to have a scale independent structure detector.

Outline of the paper. The next section deals with the Hessian matrix of a function which contains local information about its curvature. We recall how the eigenvalues of the Hessian locally describe certain structures. Then, the so-called Gauss Hessian matrix of a function obtained by smoothing the function with a Gaussian of appropriate standard deviation is revisited. Using Gaussians of different standard deviations 


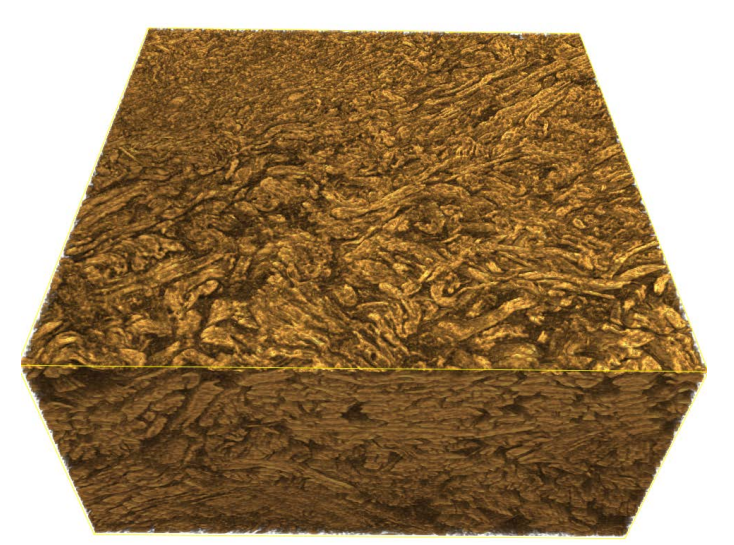

Fig. 1. Volume rendering of a $\mu C T$ image of the bovine axila leather sample studied by Bittrich et al. (2014) and Godehardt et al. (2017). Visualized are $500 \times 1000 \times 1000$ pixels corresponding to $1.6 \mathrm{~mm} \times$ $3.2 \mathrm{~mm} \times 3.2 \mathrm{~mm}$.

we obtain a multiscale structure indicator. Finally, we propose to replace the latter one by a so-called Riesz Hessian matrix which does not require a scale adaptation to the respective size of the structure. The Fourier transform, in particular the property that partial derivatives result in multiplications with the corresponding frequencies in the Fourier domain, plays a central role both in the analysis and computations. In the following section, we reconsider the local structure measures from Frangi et al. (1998). These measures can be applied for the Riesz Hessian matrix now. We present artificial as well as real-world examples demonstrating the good performance of the novel structure indicator in another section. Finally, conclusions are drawn.

\section{MATERIALS AND METHODS}

\section{HESSIAN MATRIX AS STRUCTURE TENSOR}

In this section we recall the role of the Gauss Hessian matrix as a structure tensor and introduce a Riesz Hessian matrix to cope better with structures featuring more than one scale.

\section{Hessian matrix and Fourier transform}

We start with a brief review of the Hessian matrix of a function at a point and the role of its eigenvalues for the detection of certain local structures in its graph. In general, the function has to be smoothed to cope with noise and to emphasize a special scale of its features. We recall the role of the Fourier transform in the convolution with a Gaussian and its second order derivatives.

A twice continuously differentiable function $f: \mathbb{R}^{d} \rightarrow \mathbb{R}$ can be locally approximated in a neighborhood of any $x_{0} \in \mathbb{R}^{d}$ by

$$
\begin{aligned}
f(x)=f\left(x_{0}\right) & +\nabla f\left(x_{0}\right)^{\mathrm{T}}\left(x-x_{0}\right) \\
& +\left(x-x_{0}\right)^{\mathrm{T}} \mathscr{H} f\left(x_{0}\right)\left(x-x_{0}\right) \\
& +o\left(\left\|x-x_{0}\right\|^{2}\right) .
\end{aligned}
$$

Here $\nabla f\left(x_{0}\right):=\left(\frac{\partial f}{\partial x_{j}}\left(x_{0}\right)\right)_{j=1}^{d}$ denotes the gradient of $f$ at $x_{0}$ and

$$
\mathscr{H} f\left(x_{0}\right):=\left(\frac{\partial^{2} f}{\partial x_{j} \partial x_{k}}\left(x_{0}\right)\right)_{j, k=1}^{d}
$$

its Hessian at $x_{0}$. For $r \in \mathbb{R}^{d}$, the value $r^{\mathrm{T}} \nabla f\left(x_{0}\right)$ is the directional derivative and $r^{\mathrm{T}} \mathscr{H} f\left(x_{0}\right) r$ the "second order directional derivative" of $f$ in direction $r$ at $x_{0}$. The Hessian $\mathscr{H} f\left(x_{0}\right)$ contains information on the local curvature at $x_{0}$ which can be used to identify geometrical properties of $f$ a neighborhood of $x_{0}$. More precisely, $\mathscr{H} f\left(x_{0}\right)$ is a symmetric matrix and the relation between their eigenvalues works as a tool to identify certain structures of $f$. For example, suppose that for $d=3$, the matrix $\mathscr{H} f\left(x_{0}\right)$ has the eigenvalues $\lambda_{1}\left(x_{0}\right) \leq \lambda_{2}\left(x_{0}\right) \leq \lambda_{3}\left(x_{0}\right)$. Then, the following Table 1 (bottom) from Frangi et al. (1998) gives a rough characterization of tubular, plate and blob structures in $f$. Table 1 (top) contains the information for $d=2$. In this paper, we are only interested in tubular structures. A quantitative description follows in Section "Structure indicators for tubular structures".

In applications we are rarely concerned with twice continuously differentiable functions $f$ as required in Eq. 1, but may just suppose that $f$ is in the space of (equivalence classes of) quadratic integrable functions $L_{2}\left(\mathbb{R}^{d}\right)$. However, convolution of $f$ with a $d$-variate Gaussian

$$
\varphi_{\sigma}(x):=\frac{1}{\sqrt{2 \pi \sigma^{2}} d} \exp \left(-\frac{|x|^{2}}{2 \sigma^{2}}\right)
$$

with zero mean and standard derivation $\sigma$ leads to a smooth function

$$
\left(f * \varphi_{\sigma}\right)(x)=\int_{\mathbb{R}^{d}} f(y) \varphi_{\sigma}(x-y) \mathrm{d} y .
$$

Note that for continuous functions $f \in L_{2}\left(\mathbb{R}^{d}\right)$,

$$
\lim _{\sigma \rightarrow 0}\left(f * \varphi_{\sigma}\right)(x)=f(x) \text {. }
$$


Table 1. Structures indicated by the eigenvalues of $\mathscr{H} f$, where L means low absolute value and $H$ high absolute value with the respective sign for $2 D$ (top) and $3 D$ (bottom) applications.

\begin{tabular}{cccl}
\hline$\lambda_{1}$ & $\lambda_{2}$ & & Structure \\
\hline $\mathrm{L}$ & $\mathrm{L}$ & & noise \\
$\mathrm{L}$ & $\mathrm{H}_{>0}$ & & bright tubular \\
$\mathrm{L}$ & $\mathrm{H}_{<0}$ & & dark tubular \\
$\mathrm{H}_{>0}$ & $\mathrm{H}_{>0}$ & & bright blob \\
$\mathrm{H}_{<0}$ & $\mathrm{H}_{<0}$ & & dark blob \\
\hline$\lambda_{1}$ & $\lambda_{2}$ & $\lambda_{3}$ & Structure \\
\hline $\mathrm{L}$ & $\mathrm{L}$ & $\mathrm{L}$ & noise \\
$\mathrm{L}$ & $\mathrm{L}$ & $\mathrm{H}_{>0}$ & bright plate \\
$\mathrm{L}$ & $\mathrm{L}$ & $\mathrm{H}_{<0}$ & dark plate \\
$\mathrm{L}$ & $\mathrm{H}_{>0}$ & $\mathrm{H}_{>0}$ & bright tubular \\
$\mathrm{L}$ & $\mathrm{H}_{<0}$ & $\mathrm{H}_{<0}$ & dark tubular \\
$\mathrm{H}_{>0}$ & $\mathrm{H}_{>0}$ & $\mathrm{H}_{>0}$ & bright blob \\
$\mathrm{H}_{<0}$ & $\mathrm{H}_{<0}$ & $\mathrm{H}_{<0}$ & dark blob \\
\hline & & &
\end{tabular}

From a more general point of view, the Gaussian $\varphi_{\sigma}$ is a typical function from the Schwartz space $\mathscr{S}\left(\mathbb{R}^{d}\right)$ of rapidly decreasing functions. These functions have the nice property that their derivatives as well as multiplications with polynomials are again functions in $\mathscr{S}\left(\mathbb{R}^{d}\right)$. The functions of $L_{2}\left(\mathbb{R}^{d}\right)$ are contained in $\mathscr{S}^{\prime}\left(\mathbb{R}^{d}\right)$, the space of tempered distributions, on $\mathscr{S}\left(\mathbb{R}^{d}\right)$ consisting of the linear continuous functionals $T: \mathscr{S}\left(\mathbb{R}^{d}\right) \rightarrow \mathbb{R}$ mapping $\varphi \mapsto$ $\langle T, \varphi\rangle$, see Constantin (2016). The convolution $T * \varphi$ of a function $\varphi \in \mathscr{S}\left(\mathbb{R}^{d}\right)$ with a distribution $T \in$ $\mathscr{S}^{\prime}\left(\mathbb{R}^{d}\right)$ is an infinitely often differentiable function. In particular, $f * \varphi_{\sigma}$ is infinitely often differentiable. Moreover, we have

$$
\frac{\partial^{2}}{\partial x_{j} x_{k}}(T * \varphi)(x)=\left(T * \frac{\partial^{2}}{\partial x_{j} x_{k}} \varphi\right)(x) .
$$

Finally, we will apply that the convolution is closely related to the Fourier transform. The Fourier transform $\mathscr{F}: \mathscr{S}\left(\mathbb{R}^{d}\right) \rightarrow \mathscr{S}\left(\mathbb{R}^{d}\right)$ maps $\varphi \mapsto \hat{\varphi}$ by

$$
\hat{\varphi}(\omega):=\int_{\mathbb{R}^{d}} \varphi(x) \mathrm{e}^{-2 \pi \mathrm{ix}^{\mathrm{T}} \omega} \mathrm{d} x,
$$

and its inverse is given by

$$
\varphi(x)=\int_{\mathbb{R}^{d}} \hat{\varphi}(\omega) \mathrm{e}^{2 \pi \mathrm{i} x^{\mathrm{T}} \omega} \mathrm{d} \omega .
$$

In particular, the Fourier transformed Gaussian reads

$$
\hat{\varphi}_{\sigma}(\omega)=\mathrm{e}^{-\frac{\sigma^{2}|\omega|^{2}}{2}} .
$$

The Fourier transform $\mathscr{F}: \mathscr{S}^{\prime}\left(\mathbb{R}^{d}\right) \rightarrow \mathscr{S}^{\prime}\left(\mathbb{R}^{d}\right)$ maps $T \mapsto \hat{T}$ by

$$
\langle\hat{T}, \varphi\rangle:-\langle T, \hat{\varphi}\rangle
$$

for all $\varphi \in \mathscr{S}\left(\mathbb{R}^{d}\right)$. In particular, the Fourier transform maps $L_{2}\left(\mathbb{R}^{d}\right)$ onto $L_{2}\left(\mathbb{R}^{d}\right)$. The Fourier transform has the useful property that derivatives of functions became just polynomial multiplications in the Fourier domain, i.e., for $T \in \mathscr{S}^{\prime}\left(\mathbb{R}^{d}\right)$,

$$
\left(\frac{\partial^{2}}{\partial x_{j} x_{k}} T\right)(\omega)=-(2 \pi)^{2} \omega_{j} \omega_{k} \hat{T}(\omega) .
$$

Finally, the relation between the Fourier transform and convolution is given for $\varphi \in \mathscr{S}\left(\mathbb{R}^{d}\right)$ and $T \in \mathscr{S}^{\prime}\left(\mathbb{R}^{d}\right)$ by

$$
(T * \varphi)^{\Upsilon}(\omega)=\hat{T}(\omega) \hat{\varphi}(\omega) .
$$

\section{Gauss Hessian Matrix}

Instead of the Hessian in Eq. 1 which may not exist if $f$ is not twice differentiable, we deal with the Hessian of $f * \varphi_{s}$ at $x_{0}$ for appropriate $s>0$, i.e.,

$$
\begin{aligned}
\mathscr{G} \mathscr{H} f\left(x_{0}, s\right) & :=\left(\frac{\partial^{2}}{\partial x_{j} \partial x_{k}}\left(f * \varphi_{s}\right)\left(x_{0}\right)\right)_{j, k=1}^{d} \\
& =\left(\left(f * \frac{\partial^{2}}{\partial x_{j} \partial x_{k}} \varphi_{s}\right)\left(x_{0}\right)\right)_{j, k=1}^{d}
\end{aligned}
$$

and call it Gauss Hessian matrix. Due to the decay of the second order partial derivatives of $\varphi_{s}$, the Gauss Hessian matrix can be used to detect structures which have approximately thickness $2 s$.

Note that

$$
\varphi_{\tilde{\sigma}} * \varphi_{\sigma}=\varphi_{s}, \quad s^{2}=\sigma^{2}+\tilde{\sigma}^{2} .
$$

The first and second order partial derivatives of the Gaussian $\varphi_{s}$ are given by

$$
\begin{gathered}
\frac{\partial}{\partial x_{j}} \varphi_{s}(x)=-\frac{1}{\sqrt{2 \pi s^{2} d} s^{2}} x_{j} \mathrm{e}^{-\frac{|x|^{2}}{2 \sigma^{2}}}, \\
\frac{\partial^{2}}{\partial x_{j} \partial x_{k}} \varphi_{s}(x)= \begin{cases}\frac{1}{\sqrt{2 \pi \sigma^{2}} s^{4}} x_{j} x_{k} \mathrm{e}^{-\frac{|x|^{2}}{2 s^{2}}} & \text { if } j \neq k, \\
\frac{1}{\sqrt{2 \pi \sigma^{2} d} s^{2}}\left(\frac{x_{j}^{2}}{s^{2}}-1\right) \mathrm{e}^{-\frac{|x|^{2}}{2 s^{2}}} & \text { if } j=k .\end{cases}
\end{gathered}
$$

By Eqs. 2 and 3, the second order partial derivatives appearing in the Gauss Hessian matrix can be written as

$$
\frac{\partial^{2}\left(f * \varphi_{s}\right)}{\partial x_{j} \partial x_{k}}\left(x_{0}\right)=-(2 \pi)^{2} \mathscr{F}^{-1}\left(\omega_{j} \omega_{k} \hat{f} \hat{\varphi}_{s}\right)\left(x_{0}\right) .
$$




\section{Riesz Hessian Matrix}

Instead of approximating the Hessian matrix by the Gauss Hessian matrix which can be used to detect local structures of appropriate size related to the standard deviation $s$ of the Gaussian, we will approximate it by a Riesz Hessian matrix now. This will make the detection of tubular structures less dependent on their scale and avoids computational costs due to multiscale computations.

The Riesz transform $\mathscr{R}=\left(\mathscr{R}_{j}\right)_{j=1}^{d}: L_{2}\left(\mathbb{R}^{d}\right) \rightarrow$ $\left(L_{2}\left(\mathbb{R}^{d}\right)\right)^{d}$ is defined componentwise by

$$
\begin{aligned}
\mathscr{R}_{j} f(x): & =\left(R_{j} * f\right)(x) \\
& =\underbrace{\frac{\Gamma((d+1) / 2)}{\pi^{(d+1) / 2}}}_{C_{d}} \lim _{\varepsilon \rightarrow 0} \int_{\mathbb{R}^{d} \backslash B_{\varepsilon}} \frac{y_{j} f(x-y)}{|y|^{d+1}} \mathrm{~d} y,
\end{aligned}
$$

i.e., as convolution of $f \in L_{2}\left(\mathbb{R}^{d}\right)$ with the tempered distribution

$$
R_{j}(x):=C_{d} \mathrm{pv} \cdot \frac{x_{j}}{|x|^{d+1}}, \quad j=1, \ldots, d,
$$

where $\Gamma$ denotes the Gamma function. Note that in general the convolution with of a distribution in $\mathscr{S}^{\prime}$ and a function in $L_{2}\left(\mathbb{R}^{d}\right)$ is not defined. For $d=1$, the Riesz transform coincides with the so-called Hilbert transform. In the Fourier domain the Riesz transform becomes

$$
\left(\mathscr{R}_{j} f\right)^{\Upsilon}(\omega)=\left(R_{j} * f\right)^{\prime}(\omega)=-\mathrm{i} \frac{\omega_{j}}{|\omega|} \hat{f}(\omega), \quad j=1, \ldots, d .
$$

Then

$$
\left(\mathscr{R}_{j} \mathscr{R}_{k} f\right)^{\top}(\omega)=\left(R_{j} * R_{k} * f\right)^{\top}(\omega)=-\frac{\omega_{j} \omega_{k}}{|\omega|^{2}} \hat{f}(\omega),
$$

and we recognize by Eq. 2 that

$$
\mathscr{R}_{j} \mathscr{R}_{k} f(x)=\frac{\partial^{2}}{\partial x_{j} \partial x_{k}} \Delta^{-1} f(x) .
$$

Here the inverse Laplacian is the differential operator defined by

$$
\mathscr{F}\left(\Delta^{-1} f\right)(\omega):=-\frac{1}{(2 \pi)^{2}|\omega|^{2}} \hat{f}(\omega) .
$$

In other words, $\mathscr{R}_{j} \mathscr{R}_{k} f$ can be considered as smoothed partial second order derivatives of $f$. Therefore, instead of the Gauss Hessian matrix (Eq. 4), we propose to use

$$
\mathscr{R} \mathscr{H} f\left(x_{0}, \sigma\right):=\left(\mathscr{R}_{j} \mathscr{R}_{k}\left(f * \varphi_{\sigma}\right)\left(x_{0}\right)\right)_{j, k=1}^{d}
$$

and call this Riesz Hessian matrix. In contrast to the parameter $s$ appearing in the Gauss Hessian matrix $\mathscr{G} \mathscr{H} f\left(x_{0}, s\right)$, which must be varied to detect structures at several scales, the parameter $\sigma$ in the Riesz Hessian matrix does not play a role in detecting structures of varying size. In applications, $\sigma$ will be a fixed rather small parameter to cope with the noise in images. We will see in the next section that the Riesz Hessian matrix with this fixed parameter together with the simple quantities defined there can serve for detecting structures at several scales.

To make the results comparable, we will use similarly as in Eq. 5 the Fourier transform to compute the entries of the Riesz Hessian matrix

$$
\mathscr{R}_{j} \mathscr{R}_{k}\left(f * \varphi_{\sigma}\right)=-\mathscr{F}^{-1}\left(\omega_{j} \omega_{k} \hat{f} \frac{\hat{\varphi}_{\sigma}}{(2 \pi)^{2}|\omega|^{2}}\right)\left(x_{0}\right) .
$$

\section{STRUCTURE INDICATORS FOR TUBULAR STRUCTURES}

Frangi et al. (1998) proposed measures for the detection of tubular structures for $d \in\{2,3\}$ in connection with the Gauss Hessian matrix. In the following, we recall these measures for $d=3$. Having an approximation of the Hessian based on the Riesz transform, we can also apply these measures in connection with the Riesz Hessian matrix.

By Table 1 (bottom) we have for the eigenvalues of the Gauss/Riesz Hessian matrix at a point $x \in \mathbb{R}^{3}$ lying in a tubular structure that

$$
\begin{aligned}
\lambda_{1}(x) & \approx 0, \\
\lambda_{2}(x) & \approx \lambda_{3}(x), \\
\left|\lambda_{2}(x)\right| & \approx\left|\lambda_{3}(x)\right| \gg 0,
\end{aligned}
$$

where the sign of $\lambda_{2}(x), \lambda_{3}(x)$ indicates whether it is a dark or a bright tube. To check these conditions in noisy images several quantitative indicators were introduced by Frangi et al. (1998):

The first one belongs to conditions Eq. 8 and Eq. 10 and is given by

$$
W_{a}(x):=\frac{\left|\lambda_{1}(x)\right|}{\sqrt{\left|\lambda_{2}(x) \lambda_{3}(x)\right|}} .
$$

If $W_{a}(x) \approx 0, \lambda_{1}(x) \approx 0$ and at least $\left|\lambda_{3}(x)\right| \gg 0$, it remains to check for condition Eq. 9. In this case, the value of

$$
W_{b}(x):=\left|\frac{\lambda_{2}(x)}{\lambda_{3}(x)}\right|
$$

should be close to one. With these two measures, vessels could be detected in clean images. However, in applications the data is usually affected by noise. In 
noisy regions, the Gauss Hessian has low eigenvalues, hence its Frobenius norm is small. To make the measure independent of the range and scale of the image, the maximal Frobenius norm over all image points is used as normalization. Then, a third indicator is obtained

$$
W_{c}(x):=\frac{\sqrt{\sum_{i=1}^{3} \lambda_{i}^{2}(x)}}{\max _{y} \sqrt{\sum_{i=1}^{3} \lambda_{i}^{2}(y)}} .
$$

Finally, the three measures are combined within the following product

$$
V(x, s):=\mathrm{e}^{-\frac{W_{a}^{2}(x)}{a}}\left(1-\mathrm{e}^{\frac{-W_{b}^{2}(x)}{b}}\right)\left(1-\mathrm{e}^{-W_{c}^{2}(x)}\right),
$$

where $a, b \in \mathbb{R}_{>0}$ are weights. Similar to $W_{a}$ and $W_{b}$, measures for plate- and blob-like structures can be introduced based on Table 1.

For two dimensional structures we use the following indicator

$$
V(x, s)=\mathrm{e}^{-\frac{1}{a}\left(\frac{\lambda_{1}(x)}{\lambda_{2}(x)}\right)^{2}}\left(1-\mathrm{e}^{-W_{c}^{2}(x)}\right) .
$$

Finding a fiber of thickness $2 s$ at $x$ requires a large value of $V(x, s)$. With this value of $s$, the identifier is not able to detect tubes of diameter smaller than $s$, as they are blurred too strongly by the convolution, while the tubes of thickness larger than $4 s$ are considered to be constant regions.

In practice the thicknesses of the fibers are not known, so Frangi et al. (1998) propose to search over the interval of scales $\left[s_{\min }, s_{\max }\right]$ and take

$$
\mathscr{V}(x):=\max _{s \in\left[s_{\min }, s_{\max }\right]} V(x, s)
$$

as fiber detection measure. This means that we need to calculate several Gauss Hessians for each point in the image.

We propose to relax the scale dependence of the tube indicator by using the Riesz Hessian instead of Gauss Hessian. In the following, we denote by $V_{\mathrm{G}}$, resp. $V_{\mathrm{R}}$ the measure (Eq. 11) with respect to the Gauss, resp. the Riesz Hessian, and similarly for $\mathscr{V}_{\mathrm{G}}$ and $\mathscr{V}_{\mathrm{R}}$ in Eq. 12.

\section{RESULTS}

In this section, we show how the Riesz Hessian performs in contrast to the Gauss Hessian by various artificial examples. Further, we apply the Riesz Hessian to two and three dimensional real-world data sets containing tubular structures of varying size, where we could not obtain satisfactory results using the Gauss Hessian.

\section{DISCRETIZATION}

In our experiments, we deal with discrete data $f: \mathbb{R}^{d} \supset \mathscr{G} \rightarrow \mathbb{R}$ defined on an image grid with $\mathscr{G}:=$ $\left\{1, \ldots, N_{1}\right\} \times \ldots \times\left\{1, \ldots, N_{d}\right\}, d=2,3$. To allow a comparison of the Gauss and Riesz Hessian matrix, we compute their components in the Fourier domain, i.e., by Eqs. 5 and 7, respectively. The computation of the discrete Fourier transforms is realized using the function $f f t n$ of MATLAB. We mirror the image at the boundary for the calculation of the Hessians, to avoid artifacts occurring from the periodicity assumption of the discrete Fourier transform. Hence, for the calculation of the Hessian matrices, we consider the image $\tilde{f}:\left\{1, \ldots, 2 N_{1}\right\} \times \ldots \times\left\{1, \ldots, 2 N_{d}\right\} \rightarrow \mathbb{R}$ with

$$
\tilde{f}(x)=f(\min \{x, 2 N+1-x\}),
$$

where $N:=\left(N_{i}\right)_{i=1}^{d}$ and $\min$ is meant componentwise.

\section{ARTIFICIAL 2D DATA}

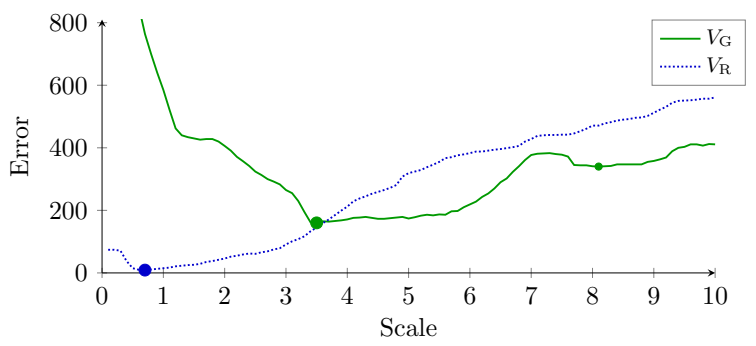

Fig. 2. Error plot of structure indicators based on Gauss and Riesz Hessians with threshold 0.15(1$\left.\mathrm{e}^{-1}\right)$.

The artificial fork-like structure in Fig. 3a has vessels in two different scales. The image is of size $55 \times 55$ pixels with values in $[0,1]$. The thick vessel is 15 pixel wide and the thin one 5. It is corrupted by additive Gaussian noise of standard deviation 0.3. We apply the indicator $V_{\bullet}(x, s), \bullet \in\{\mathrm{G}, \mathrm{R}\}$ defined in Eq. 11 with $a:=1$ for different scales $s$ to the noisy image. We assume that a pixel $x \in \mathscr{G}$ belongs to the tubular structure, if $V_{\bullet}(x, s) \geq \delta\left(1-\mathrm{e}^{-1}\right)$, where $\delta:=0.15$. Note that $1-\mathrm{e}^{-1}$ is the maximal possible value $V_{\bullet}$ can achieve. As error measure we use the number of falsely classified pixels. Fig. 2 shows the error plot based on $V_{\bullet}, \bullet \in\{\mathrm{G}, \mathrm{R}\}$ for different scales. The Riesz Hessian method produces the smallest error for $s=0.7$. This confirms the fact that the convolution is only necessary for smoothing the noise. Fig. 3c depicts the very good result. The Gauss Hessian approach has a larger error and we recognize a global minimum at $s=3.5$ and a local minimum at $s=8.1$. 

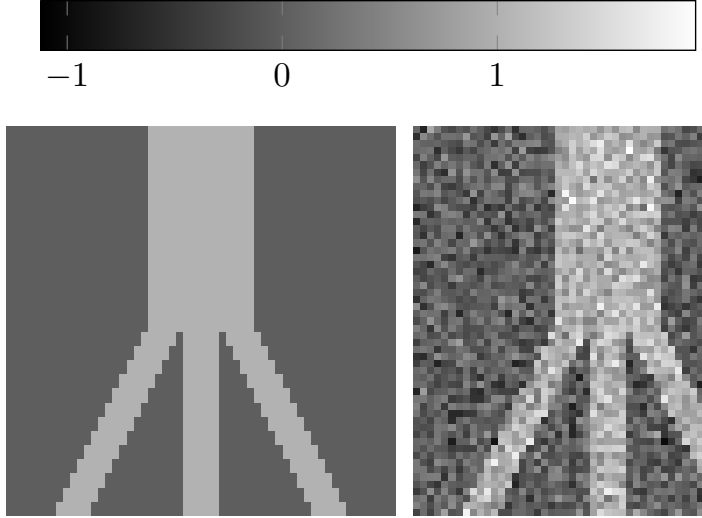

(a) Original fork
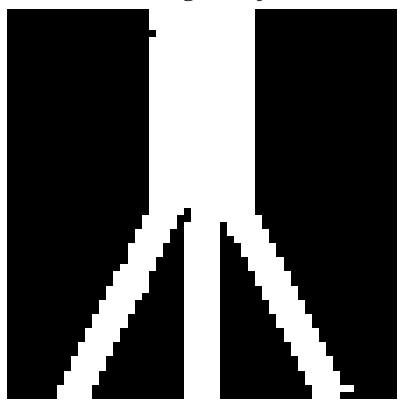

(c) $V_{\mathrm{R}}$ for $s=0.7$

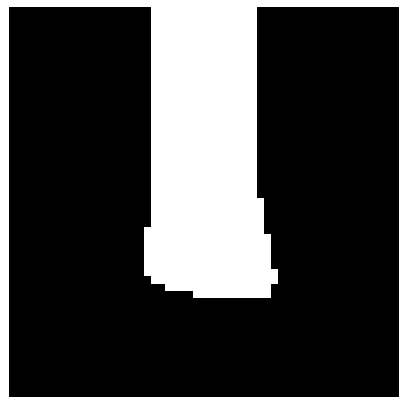

(e) $V_{\mathrm{G}}$ for $s=8.1$

Fig. 3. Results of structure indicators based on Gauss and Riesz Hessians applied to an artificial noisy 2D fork with white Gaussian noise $(\sigma=0.3)$. (a),(b) are represented as gray values according to the above color map, (c)-(f) white indicates a detected structure and black means no detection.

These minima correspond to the detection of the different vessel sizes. In Fig. 3d, we see that for $s=$ 3.5, small vessels are well recognized, except for the branching point, while the large vessel is estimated too thin. Using $\sigma=8.1$ the large vessel is detected well, while the branching point is over-smoothed and the small vessels disappear, see Fig. 3e. Instead of $V_{\bullet}(x, s)$ at different scales we could use $\mathscr{V}_{\bullet}$, i.e., take the maximum over the interesting scales. To find these scales we perform a grid search on $\frac{1}{10} \mathbb{N}$ for all possible combinations of $s_{\min }<s_{\max } \in\left[\frac{1}{10}, 10\right]$, as well as for the best percentage $\delta$ of $1-\mathrm{e}^{-1}$ in $\frac{1}{100} \mathbb{N}$ as

threshold. The optimal parameters and corresponding errors are reported in Table 3. In contrast to the Gauss Hessian approach, the result for the Riesz Hessian improves only slightly. However, as shown in Fig. 3f, the larger scale part of the fork is over-smoothing at the branching point.

Table 2. Optimal parameters and related errors for $V_{\bullet}$.

\begin{tabular}{crr}
\hline Indicator & $s_{\mathrm{Opt}}$ & Error \\
\hline$V_{\mathrm{G}}$ & 3.5 & 160 \\
$V_{\mathrm{R}}$ & 0.7 & 9 \\
\hline
\end{tabular}

Table 3. Optimal parameters and related errors for $\mathscr{V}_{\bullet}$.

\begin{tabular}{clllr}
\hline Indicator & $s_{\min }$ & $s_{\max }$ & $\delta$ & Error \\
\hline $\mathscr{V}_{\mathrm{G}}$ & 1.0 & 9.9 & 0.31 & 95 \\
$\mathscr{V}_{\mathrm{R}}$ & 0.6 & 0.8 & 0.16 & 7 \\
\hline
\end{tabular}

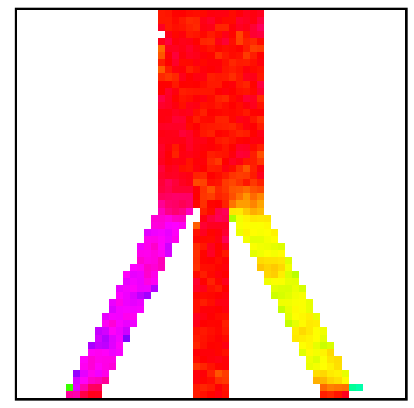

(a) Angle of $e_{1, \mathrm{R}}, s=0.7$

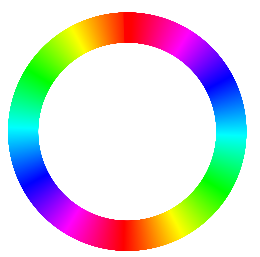

(c) Color coding

Fig. 4. Directions of the detected tubular structures given by the direction of the eigenvector of the smallest eigenvalue of the Hessian.

We are not only interested in the position of the structures but also in their directions. This can be done by considering the normalized eigenvector $e_{1}$ of the smallest eigenvalue of the corresponding Hessian. In Fig. 4, we show the angles of the eigenvectors at points, where a structure is detected. The detected directions coincide in most parts with those of the tubular structure, except for the bifurcation with the Gauss Hessian approach. However, there are some errors in the directions detected via the Gauss Hessian 
due to smooth direction changes. Note that due to the mirror boundary conditions, the angles are slightly disturbed at the bottom.

\section{SPHEROIDAL GRAPHITE IN GRAY CAST IRON}

Cast iron with spheroidal graphite is also known as ductile cast alloy and is popular due to its good ductile behavior. The spherical graphite inclusions yield better energy absorption during loading and thus higher ultimate tensile strength characteristics are reached. Fig. 5(a) shows a micrograph of cast iron with spheroidal graphite where we see a section through the spherical inclusions and grain boundaries of the polycrystalline iron matrix.

Our approach can be used to detect the spheroidal graphite inclusions. For blobs in two dimensions we use the following structure indicator

$$
V(x, s)=\left(1-\mathrm{e}^{-\frac{1}{a}\left(\frac{\lambda_{1}(x)}{\lambda_{2}(x)}\right)^{2}}\right)\left(1-\mathrm{e}^{-W_{c}^{2}(x)}\right)
$$

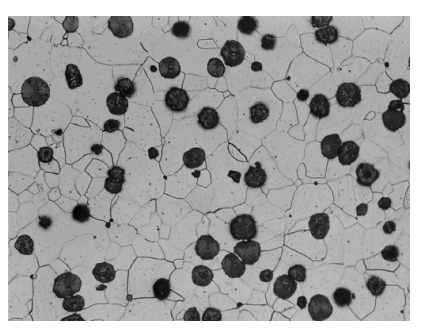

(a) original
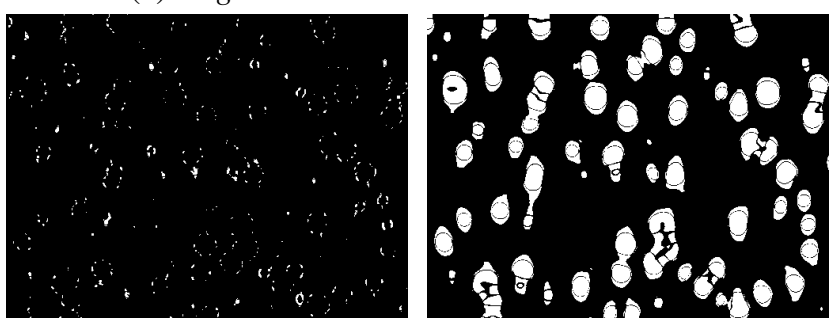

(b) small blobs Gauss,

(c) big blobs Gauss,

$s=2.0, \delta=0.2, a=0.01$

$s=5.0, \delta=0.05, a=0.01$

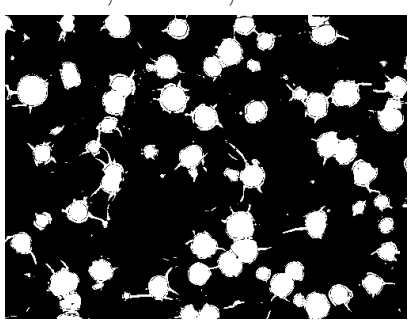

(d) blobs Riesz

$\sigma=0.8, \delta=0.2, a=0.01$

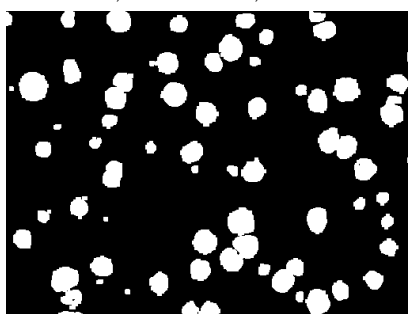

(e) blobs Riesz,

morphologically opened

Fig. 5. 2D blob detection illustrated using the example of spheroidal graphite in gray cast iron. The structuring element for the morphological opening is a square of edge length 5 pixels. and use as threshold $\delta\left(1-\mathrm{e}^{-1}\right)^{2}$. The results in Fig. 5 show that the Riesz Hessian is able to detect both small and large structures in one detection step. Moreover, large blobs that are close together are separated cleaner even without postprocessing. Note that the postprocessing is performed on binary data and is very fast.

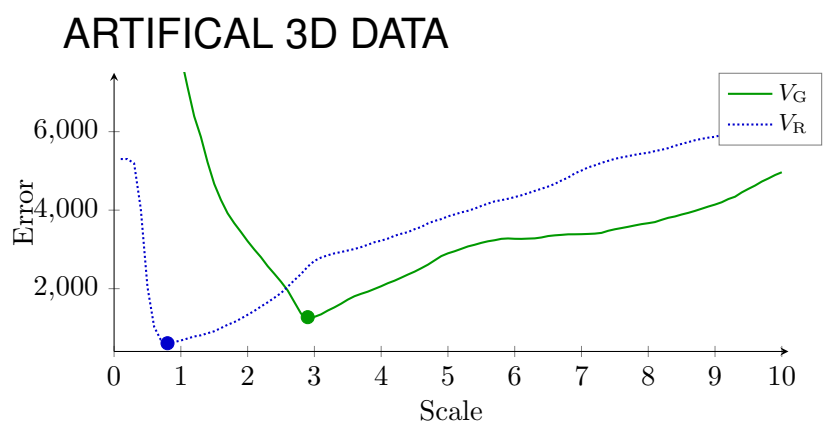

Fig. 6. Error plot of structure indicators based on Gauss and Riesz Hessians with threshold 0.02(1 $\left.\mathrm{e}^{-1}\right)^{2}$.

Next we consider the 3D fork like structure corrupted by Gaussian noise with standard deviation $\sigma=0.5$ in Fig. 7. The original image is of size $35 \times 35 \times 35$, the thick tube of $15 \times 15$ and the thin one of $5 \times 5$ pixels. The tubular structures are detected if the indicator $V_{\bullet}$ is larger than $\left(1-\mathrm{e}^{-1}\right)^{2} / 50$, i.e., a value larger than $2 \%$ of the maximal response value. Fig. 6 shows the errors for different scales. The optimal scales with the corresponding errors is given in Table 4. The Riesz Hessian yields the best result for small $s=0.8$. The Gauss Hessian performs worse even at the best scale $s=2.9$. The tubular structures indicated by $V_{\bullet}$ at the optimal scales are shown in Fig. 7d and c. The result based on the Gauss Hessian looks very blurry and detects background areas as structures. The structures detected by the Riesz Hessian approach fit the original data much better. Still, some small areas of the background are considered to belong to the big tube. The indicator $\mathscr{V}_{\bullet}$ in Table 5, does not give an improvement for the Riesz Hessian method, but the Gauss Hessian approach profits from the different scales. However, the interval $\left[s_{\min }, s_{\max }\right]$ is smaller than in two dimensions and does not include scales corresponding to the large tube. The result is visualized in Fig. 7e. It has sharper edges as those obtained via $V_{\mathrm{G}}(\cdot, 2.9)$, but more background is classified wrongly.

Table 4. Optimal parameters and related errors for $V_{\bullet}$.

\begin{tabular}{ccl}
\hline Indicator & $s_{\mathrm{opt}}$ & Error \\
\hline$V_{\mathrm{G}}$ & 2.9 & 1274 \\
$V_{\mathrm{R}}$ & 0.8 & 610 \\
\hline
\end{tabular}




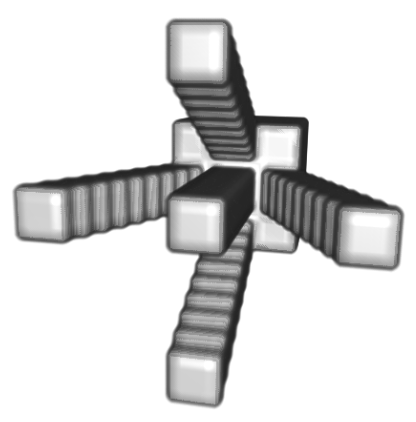

(a) Original $3 D$ fork

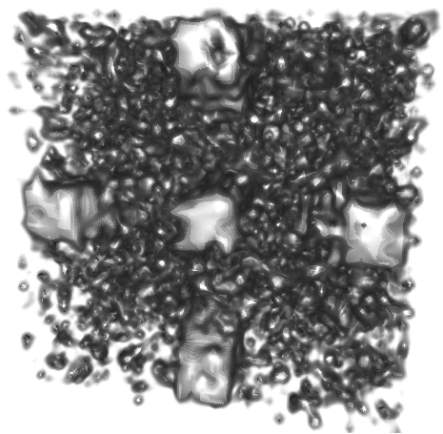

(b) Noisy $3 D$ fork

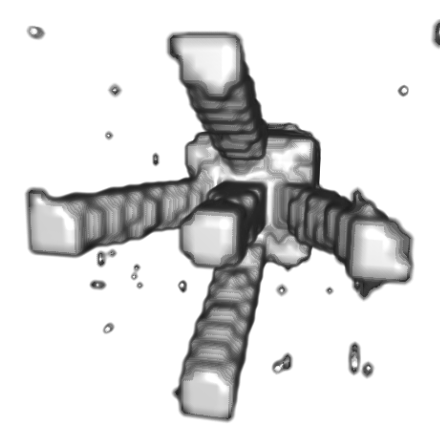

(c) $V_{\mathrm{R}}$ for $s=0.8$

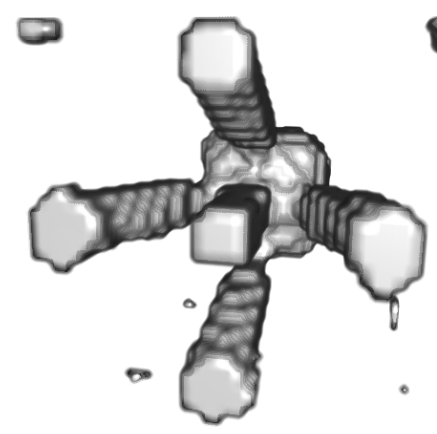

(d) $V_{\mathrm{G}}$ for $s=2.9$

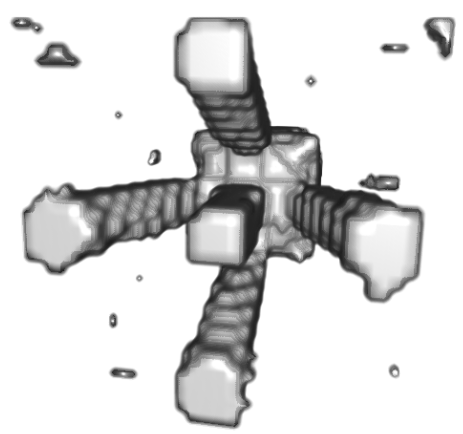

(e) $\mathscr{V}_{\mathrm{G}}$

Fig. 7. Results of structure indicators based on Gauss and Riesz Hessians applied to an artificial noisy 3D fork with white Gaussian noise $(\sigma=0.5)$.

Table 5. Optimal parameters and related errors for $\mathscr{V}_{\bullet}$

\begin{tabular}{cllll}
\hline Indicator & $s_{\min }$ & $s_{\max }$ & $\delta$ & Error \\
\hline $\mathscr{V}_{\mathrm{G}}$ & 2 & 3 & 0.03 & 1129 \\
$\mathscr{V}_{\mathrm{R}}$ & 0.8 & 0.8 & 0.02 & 610 \\
\hline
\end{tabular}

We are also interested in the directions of the tubular structures, which are detected by the different methods. We show the directions of the eigenvectors corresponding to the smallest eigenvalue at each detected point in Fig. 8. The colorization is based on the angle of the eigenvector to the $x$-axis in the $x y$ plane. In the thin tubular structure both methods lead to similar estimates of the directions, which match the direction of the fibers. The Gauss Hessian method does not detect the whole tube near the branching point. Both methods find wrongly classified directions in the background. This observation corresponds to the detection seen in Fig. 7.

Fig. 9 shows two slices of the fork, one from the thick tube and the other close to the tip, i.e., at $z=10,30$. In contrast to the Gauss Hessian method, the Riesz Hessian one detects the whole rectangular structure of the thick tube, but the directions at the left and right corner are slightly disturbed.

\section{LOCAL 3D FIBER ORIENTATION ANALYSIS FOR FIBROUS MATERIALS}

Here, we apply the orientation analysis based on the Gauss and Riesz Hessian to a synchrotron radiation $\mu \mathrm{CT}$ (SR $\mu \mathrm{CT})$ of a sample from a felt used for dewatering the pulp during paper production. This data set generated at the ESRF in Grenoble (Cloetens et al., 2002) has been used to study flow properties (Rief, 2005), see also Ohser and Schladitz (2009, Section 3.4). Fig. 10 shows the volume rendering of a $590 \times 600 \times 450$ pixel subsample clearly featuring thin and thick fibers. We illustrate the potential of replacing the gradients by the Riesz transform for simultaneous orientation analysis of fiber systems with varying fiber thickness. 


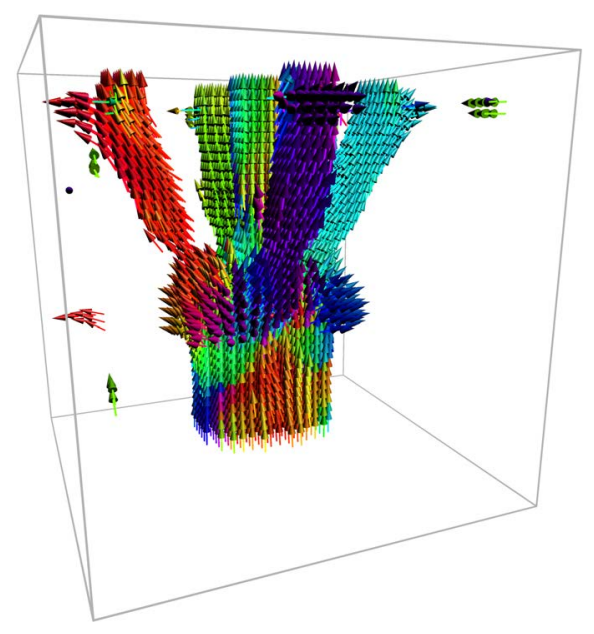

(a) $e_{1, \mathrm{G}}$ for $s=2.8$

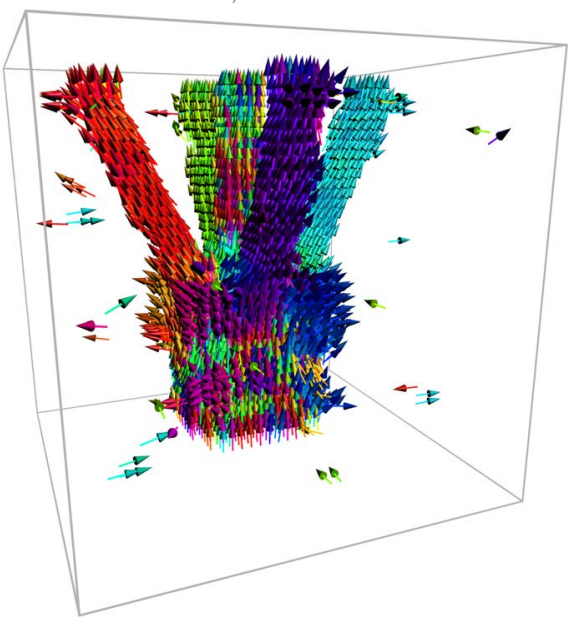

(b) $e_{1, \mathrm{R}}$ for $s=0.8$

Fig. 8. Directions of the detected tubular structures given by the direction of the eigenvector of the smallest eigenvalue of the Hessian.

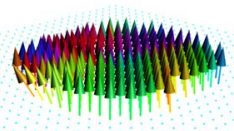

(a) $e_{1, \mathrm{G}}$ for $s=2.8$

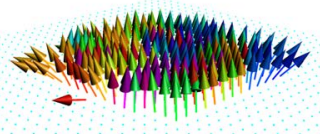

(c) $e_{1, \mathrm{R}}$ for $s=0.8$

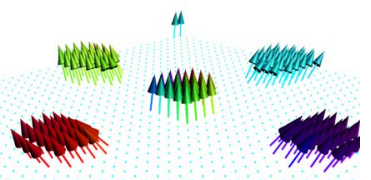

(b) $e_{1, \mathrm{G}}$ for $s=2.8$

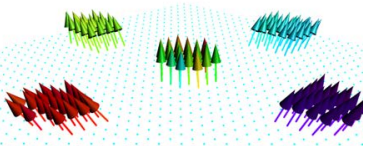

(d) $e_{1, \mathrm{R}}$ for $s=0.8$
Fig. 9. Slices trough the thick and thin part of the tubes shown in Fig. 8.

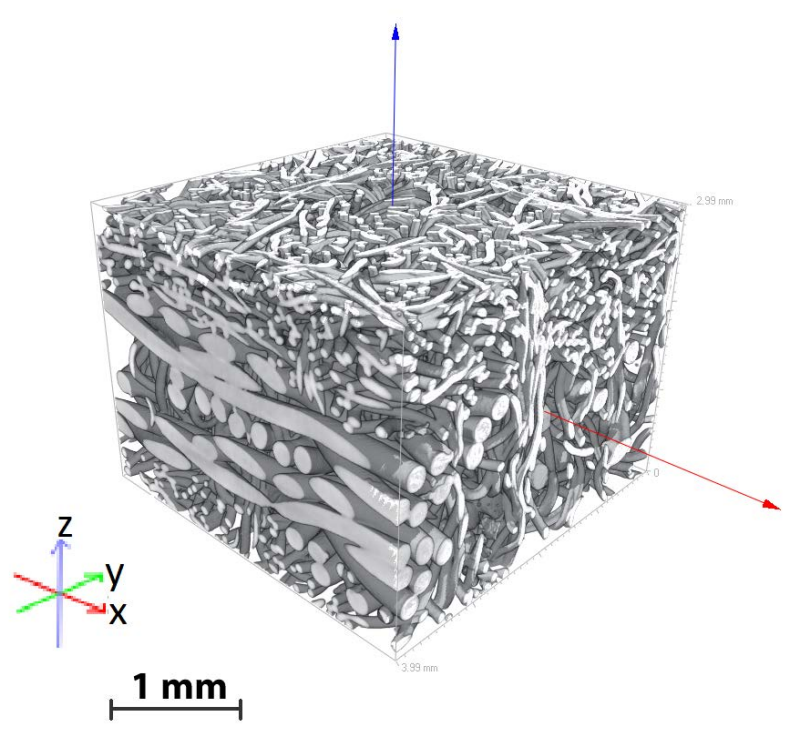

Fig. 10. Fiber felt sample, $590 \times 600 \times 450$ subvolume of the SR $\mu C T$ data set. Volume rendering of the original data set.

Visual impression suggests that the thick fibers are mainly oriented in the $x$-direction. The analysis based on the Gauss Hessian with $\sigma=5$ does not capture all thick fibers properly, see Fig. 11a. The analysis based on the Gauss Hessian with $\sigma=15$ smudges both structure and local orientation results, see Fig. 11b. The Hessian based on the Riesz transform however, finds very consistently both thin and thick fibers in $x$-direction, see Fig. 11c and the orientation histogram 11e.

To summarize, for real CT image data of structures with strongly varying fiber thicknesses, the Riesz transform based method yields better local orientation results than those obtained by the state-of-the-art method. The fact, that it does not rely on the prior knowledge about the observed fiber thickness, is a huge advantage in all applications where fibers of varying thickness have to be analyzed.

\section{SEGMENTATION OF TYPICAL STRUCTURAL ELEMENTS OF BOVINE LEATHER}

The simulation of mechanical properties of bovine leather is of great practical interest, but needs a deep understanding of the microstructure. In particular, the segmentation of the collagen fibers is essential. The method consists of three basic steps: first, the original data set is filtered with a median and a soft shock filter to get rid of noise and to obtain a basic 


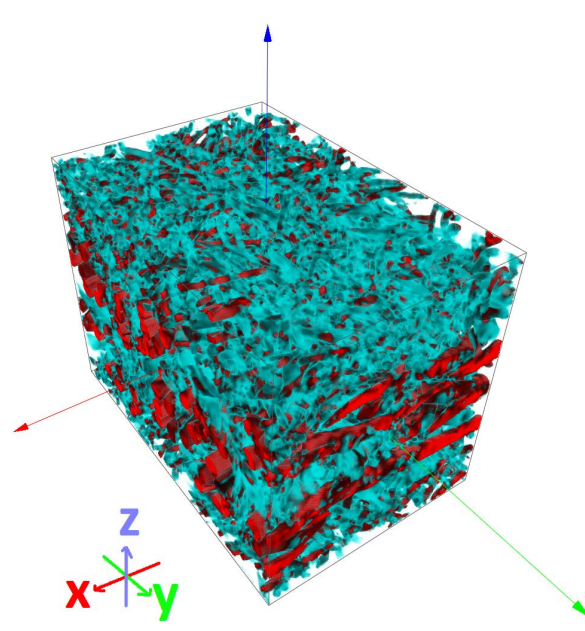

(a) $\delta_{x x}$, Gauss Hessian, $\sigma=5$

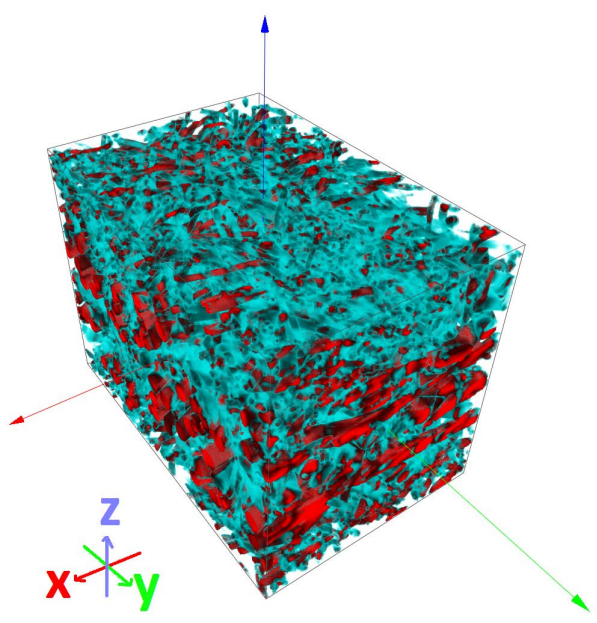

(c) $\delta_{x x}$, Riesz Hessian

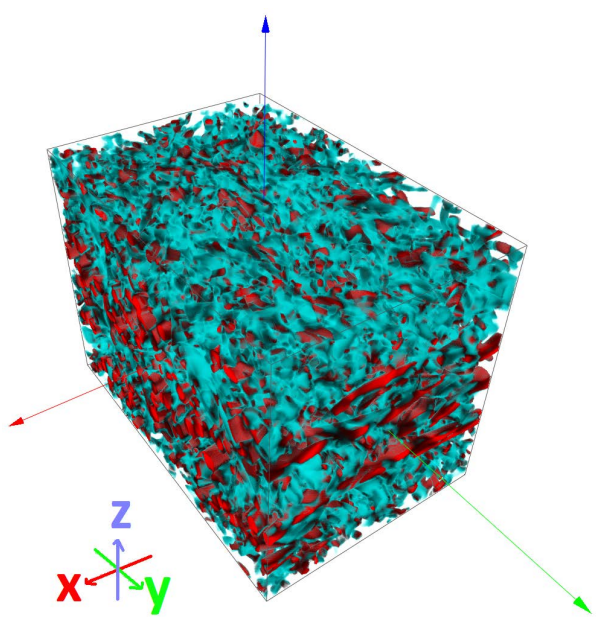

(b) $\delta_{x x}$, Gauss Hessian, $\sigma=15$

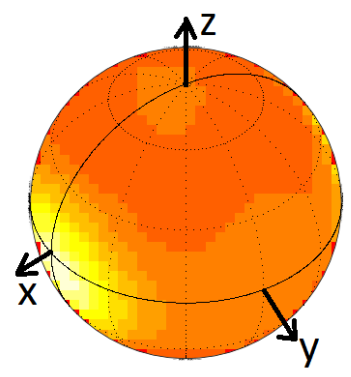

(e) $(\hat{\phi}, \hat{\theta})$-histogram, Riesz Hessian

Fig. 11. Fiber orientation analysis results for fiber felt sample. The visualized results are clipped down in positive $x$-direction from originally 590 slices to 400 in order to oppress the boundary effects. $(a),(b),(c) x x$-components $\delta_{x x}$ of the computed Hessians. (a) Gauss Hessian using $\sigma=5$ to match thin fibers. (b) Gauss Hessian using $\sigma=15$ to match thick fibers. (c) Riesz Hessian. (d) Color legend for $(a),(b),(c)$. (e) Volume weighted histogram of the local fiber orientations for method (c).

segmentation into fore- and background. From the denoised data we want to compute normal directions, because they are very sensitive to fiber boundaries, i.e., they change a lot at the fiber boundaries. To obtain a robust estimation of the normal direction the Riesz Hessian matrix is computed. Note that the local fiber bundle thickness in the leather varies strongly, such that it is impossible to choose the right scale $s$ for calculating the derivatives by the Gauss Hessian. Subsequently, the local normal directions are derived as those corresponding to the largest eigenvalue of the Riesz Hessian and the local differences of these normal directions are calculated, $c f$. Fig. 12b. Finally, the watershed transform, $c f$. Fig. 12c, and a hierarchical coarsening (Beucher, 1994) is applied to the thus derived orientation gradient image. The latter thrives on differences of features of the existing regions. A slice of the final segmentation is shown in Fig. 12d. This result can now be used to analyze the microstructure of the leather, see Godehardt et al. (2017). So far, this is the only successful attempt on automatic segmentation of individual fiber bundles from CT images of leather samples. Note that the method was also reported without giving the mathematical details in this short conference paper.

\section{DISCUSSION}

In this paper, we modified the Gauss Hessian by replacing the derivatives of the Gaussian smoothed function in Eq. 4 with the respective Riesz 

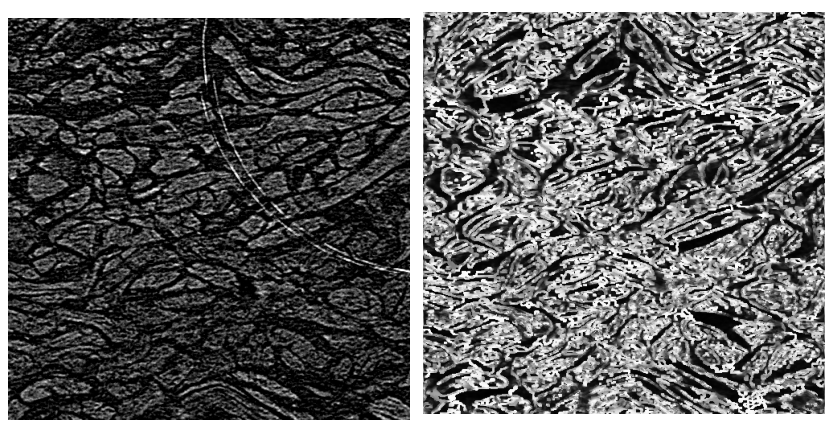

(a) original

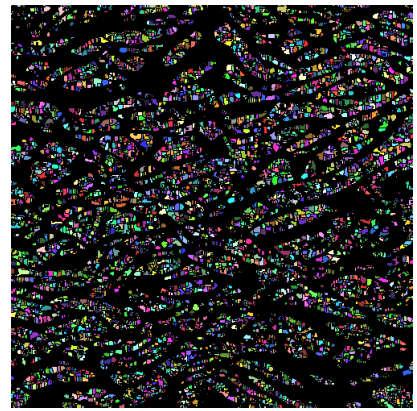

(c) initial segmentation (b) local gradient of normal orientation

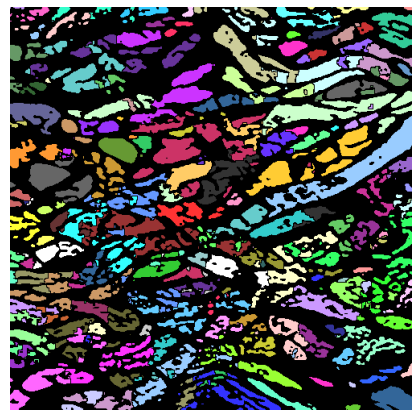

(d) final segmentation

Fig. 12. Slices through the leather data set "axila" from Bittrich et al. (2014), steps of the segmentation. The initial segmentation is achieved by the watershed transform on the orientation gradient image as described above, the final one after four waterfall coarsening steps. See Godehardt et al. (2017) for details.

transforms in Eq. 6. As a result, both the local orientation analysis as well as Frangi's local structure indicators based on the Riesz Hessian matrix are less restrictive to the scale of the tubular structures. The fact, that they do not rely on the prior knowledge about the observed fiber thickness, is a big advantage in all applications, where fibers of varying thickness have to be analyzed. This was demonstrated by various numerical examples including artificial test images as well as real world applications in two and three dimensions. Even though in most examples the focus was on the detection of tubular structures, the Frangi Riesz Hessian indicators can be used for plate or blob detection as well.

\section{ACKNOWLEDGMENTS}

This work was supported by the German Federal Ministry of Education and Research through projects 05M2013 (AniS) and 05M16RCA (AMSCHA).

\section{REFERENCES}

Aach T, Mota C, Stuke I, Mühlich M, Barth E (2006). Analysis of superimposed oriented patterns. IEEE T Image Process 15:3690-700.

Altendorf H, Jeulin D (2009). 3D directional mathematical morphology for analysis of fiber orientations. Image Anal Stereol 28:143-53.

Basil-Jones MM, Edmonds RL, Allsop TF, Cooper SM, Holmes G, Norris GE, Cookson DJ, Kirby N, Haverkamp RG (2010). Leather structure determination by small-angle X-ray scattering (SAXS): Cross sections of ovine and bovine leather. J Agr Food Chem 58:528691.

Beucher S (1994). Watershed, hierarchical segmentation and waterfall algorithm. In: Serra J, Soille P, eds., Computational imaging and vision Vol. 2: Mathematical morphology and its applications to image processing. Dordrecht: Kluwer, pp 69-76.

Bittrich E, Schladitz K, Meyndt R, Schulz H, Godehardt M (2014). Micro-computed tomography studies for threedimensional leather structure analysis. J Am Leather Chem Assoc 109:367-71.

Cloetens P, Ludwig W, Boller E, Helfen L, Salvo L, Mache R, Schlenker M (2002). Quantitative phase contrast tomography using coherent synchrotron radiation. Proc SPIE 4503:82-91.

Constantin A (2016). Fourier analysis, Vol 1: Theory. Cambridge: Cambridge University Press.

Du YP, Parker DL, Davis WL (1995). Vessel enhancement filtering in three-dimensional MR angiography. J Magn Reson Imaging 5:353-9.

Felsberg M, Granlund G (2004). POI detection using channel clustering and the 2D energy tensor. In: Rasmussen CE, Bülthoff HH, Schölkopf B, Giese MA, eds., Pattern recognition: Proc 26th DAGM Symp. Lect Not Comput Sci 3175:103-10.

Felsberg M, Sommer G (2001). The monogenic signal. IEEE T Signal Process 49:3136-44.

Förstner W, Gülch E (1987). A fast operator for detection and precise location of distinct points, corners and centres of circular features. In: Proc ISPRS Intercommiss Conf Fast Process Photogramm Data, pp 281-305.

Frangi AF, Niessen WJ, Vincken KL, Viergever MA (1998). Multiscale vessel enhancement filtering. In: Wells WM, Colchester A, Delp S, eds. Medical image computing and computer-assisted interventation (MICCAI'98). Lect Not Comput Sci 1496:130-7.

Godehardt M, Schladitz K, Dietrich S, Meyndt R, Schulz $H$ (2017). Segmentation of collagen fiber bundles in 3D by waterfall on orientations. In: Angulo J, VelascoForero S, Meyer F, eds. Mathematical morphology and its applications to signal and image processing: Proc 13th Int Symp (ISMM 2017). Lect Not Comput Sci 10225:447-54.

Häuser S, Heise B, Steidl G (2014). Linearized Riesz 
transform and quasi-monogenic shearlets. Int $\mathbf{J}$ Wavelets Multi 12:1450027.

Heieck F, Hermann F, Middendorf P, Schladitz K (2017). Influence of the cover factor of 2D biaxial and triaxial braided carbon composites on their in-plane mechanical properties. Compos Struct 163:114-22.

Köthe U (2003). Edge and junction detection with an improved structure tensor. In: Michaelis B, Krell G, eds. Pattern recognition: Proc 25th DAGM Symp. Lect Not Comput Sci 2781:25-32.

Köthe U, Felsberg M (2005). Riesz-transforms versus derivatives: On the relationship between the boundary tensor and the energy tensor. In: Kimmel R, Sochen NA, Weickert J, eds. Scale space and PDE methods in computer vision: Proc 5th Int Conf. Lect Not Comput Sci 3459:179-91.

Krause M, Hausherr J, Burgeth B, Herrmann C, Krenkel W (2010). Determination of the fibre orientation in composites using the structure tensor and local X-ray transform. J Mater Sci 45:888-96.

Larkin KG, Bone DJ, Oldfield MA (2001). Natural demodulation of two-dimensional fringe patterns: I. general background of the spiral phase quadrature transform. J Opt Soc Am A 18:1862-70.

Lindeberg T (1999). Principles for automatic scale selection. In: Handbook on Computer Vision and Applications, vol. 2. Academic Press.

Ohser J, Schladitz K (2009). 3D Images of materials structures - Processing and analysis. Weinheim: Wiley $\mathrm{VCH}$.

Prince MR (1994). Gadolinium-enhanced MR aortography. Radiology 191:155-64.

Redenbach C, Rack A, Schladitz K, Wirjadi O, Godehardt M (2012). Beyond imaging: on the quantitative analysis of tomographic volume data. Int J Mater Res 2:217-27.

Rief S (2005). Nonlinear flow in porous media-numerical solution of the Navier-Stokes system with two pressures and application to paper making: $\mathrm{PhD}$ thesis. Kaiserslautern: Technische Universität Kaiserslautern.

Riesz M (1928). Sur les fonctions conjuguées. Math Z 27:218-44.

Robb K, Wirjadi O, Schladitz K (2007). Fiber orientation estimation from 3D image data: Practical algorithms, visualization, and interpretation. In: Proc 7th Int Conf Hybrid Intel Syst (HIS 2007). pp 320-5.
Schausberger SE, Heise B, Maurer C, Bernet S, RitschMarte M, Stifter D (2010). Flexible contrast for lowcoherence interference microscopy by Fourier-plane filtering with a spatial light modulator. Opt Lett 35:4154-6.

Sedlazeck A (2008). Local feature detection by higher order Riesz transforms on images: Diplomarbeit. Kiel: Christian-Albrechts-Universität.

Sliseris J, Andrä H, Kabel M, Wirjadi O, Dix B, Plinke B (2016). Estimation of fiber orientation and fiber bundles of MDF. Mater Struct 49:4003-12.

Steidl G, Teuber T (2009). Anisotropic smoothing using double orientation. In: Lie A, Lysaker M, Morken K, Tai XC, eds. Proc 2nd Int Conf Scale Space Meth Variat Meth Comput Vision (SSVM 2009). Lect Not Comput Sci 5567:477-89.

Stein EM, Weiss GL (1971). Introduction to Fourier analysis on Euclidean spaces, vol 1. Princeton University Press.

Storath M (2011). Directional multiscale amplitude and phase decomposition by the monogenic curvelet transform. SIAM J Imaging Sci 4:57-78.

Unser M, Van De Ville D (2010). Wavelet steerability and the higher-order Riesz transform. IEEE T Image Proces 19:636-52.

Wells HC, Holmes G, Haverkamp RG (2016). Looseness in bovine leather: microstructural characterization. J Sci Food Agr 96:2731-6.

Wirjadi O, Godehardt M, Schladitz K, Wagner B, Rack A, Gurka M, Nissle S, Noll A (2014). Characterization of multilayer structures in fiber reinforced polymer employing synchrotron and laboratory X-ray CT. Int J Mater Res 105:645-54.

Wirjadi O, Schladitz K, Easwaran P, Ohser J (2016). Estimating fibre direction distributions of reinforced composites from tomographic images. Image Anal Stereol 35:167-79.

Wirjadi O, Schladitz K, Rack A, Breuel T (2009). Applications of anisotropic image filters for computing 2D and 3D-fiber orientations. In: Stereology and Image Analysis: Proc 10th Eur Congr ISS (ECS 09), pp 10712.

Wonisch A, Wüst A (2014). More precise part design: Accurate simulation of fiber orientation of glass fiberreinforced plastics. Kunststoffe Int 9:80-3. 